\title{
Low temperature synthesis of ZnSe nanowires by self-catalytic liquid-solid growth
}

\author{
Chung-Liang Cheng, Yang-Fang Chen* \\ Department of Physics, National Taiwan University, Taipei 10617, Taiwan
}

\section{A R T I C L E I N F O}

\section{Article history:}

Received 29 July 2008

Received in revised form 15 October 2008

Accepted 16 November 2008

\section{Keywords:}

Nanostructures

Semiconductors

Chemical vapour deposition (CVD)

$\mathrm{X}$-ray scattering

Luminescence

\begin{abstract}
A B S T R A C T
Nanowire arrays of zinc selenide with zinc blende structures have been synthesized on the zinc foil via a simple thermal evaporation route at relatively low temperature $\left(\sim 400^{\circ} \mathrm{C}\right)$. A self-catalytic liquid-solid growth is proposed for the formation of the nanowires. The nanowires are usually $50-150 \mathrm{~nm}$ in diameter, and several microns in length. Room-temperature cathodoluminescence spectrum from zinc blende $\mathrm{ZnSe}$ nanowires shows a near band edge emission band peaked at around $462 \mathrm{~nm}$ and a broad deep-level emission band at around $580 \mathrm{~nm}$, respectively.
\end{abstract}

(c) 2008 Elsevier B.V. All rights reserved.

\section{Introduction}

Zinc selenide (ZnSe), which has a direct wide band gap of $2.7 \mathrm{eV}$ and a large exciton binding energy of $21 \mathrm{meV}$, has attracted intensive attention for its applications such as blue light-emitting devices and laser diodes. Recently, a variety of nanostructured ZnSe materials including nanorods [1], nanoribbons [2], nanowheels [3], nanorings [4], nanopowders [5], and nanowires [6,7] have been reported due to their potential applications in constructing nanoscale optoelectronic devices.

The preparation of $\mathrm{ZnSe}$ nanostructures has been demonstrated by various methods, including vapour phase methods [8], hydrothermal process [9], and template-based methods [10]. Among them, vapour phase methods such as thermal evaporation and condensation [3,11], and evaporation of organic zinc precursors [7] have been used for producing ZnSe nanostructures. However, such methods often use metal catalysts such as Au $[3,12]$ to assist and control the growth process. Hence, the remains of metals will influence the purity of the final products. Therefore, seeking self-catalytic growth techniques of $\mathrm{ZnSe}$ nanowires is important.

In this paper, we report the synthesis of ZnSe nanowire arrays on the zinc foil by the incorporation of selenium powder without using any other metal catalysts. The ZnSe nanowire arrays were char-

\footnotetext{
* Corresponding author at: No. 1, Section 4, Roosevelt Road, Taipei, Taiwan. Tel.: +8862 33665125; fax: +886223639984.

E-mail address: yfchen@phys.ntu.edu.tw (Y.-F. Chen).
}

acterized by different measurements. Meanwhile, possible growth mechanism of ZnSe nanowires was discussed.

\section{Experimental details}

In a typical procedure, selenium powder and a zinc foil $(8 \mathrm{~mm} \times 10 \mathrm{~mm} \times$ $0.25 \mathrm{~mm}$ ) were kept in an alumina boat (the zinc foil was placed $1 \mathrm{~cm}$ downstream the selenium powder) and placed at the center of a quartz tube which was inserted into a horizontal tube furnace along the argon gas flow direction in sequence. The reaction chamber was sealed and pumped to and keep at a pressure 200 Torr throughout the experiment. Argon (purity: 99.9\%) was introduced into the reaction chamber at a flow rate of 100 standard cubic centimetres per minute $(\mathrm{sccm})$ as a carrier gas. Subsequently, the temperature of furnace was raised to $400{ }^{\circ} \mathrm{C}$ at a rate of $40{ }^{\circ} \mathrm{C} \mathrm{min}^{-1}$ and maintained at this temperature for $1.5 \mathrm{~h}$. Finally, it was cooled to room temperature. After reaction, the products grew on the surface of the zinc foil as yellow films. There was still rest of Se vapour which did not react with zinc in the chamber. In order to collect the rest of the Se vapour, we constructed a homemade cold-trap system to solidify Se vapour in the downstream zone. After reaction, we can clearly see that the rest of the Se vapour was solidified and became grayblack solid Se bulk in the downstream zone. Hence, we can minimize the Se vapour pollution in the environment.

The morphology of as-prepared products was determined by field emission SEM (FE-SEM, JSM-6500F) equipped with EDX spectrometer. X-ray diffraction (XRD) patterns were obtained on a PANalytical X'Pert Pro diffracometer using $\mathrm{Cu} K \alpha$ radiation $(\lambda=1.5418 \AA$ ) at $45 \mathrm{kV}$ and $40 \mathrm{~mA}$. Raman scattering spectrum was recorded using a Jobin-Yvon T64000 Raman-scattering spectrometer at room temperature in the backscattering geometry. The $514.5 \mathrm{~nm}$ line of a $100 \mathrm{~mW} \mathrm{Ar}^{+}$laser was used for the excitation source. The excitation power was $160 \mathrm{~W} \mathrm{~cm}^{-2}$ and the spot size of the laser light was focused to about $40 \mu \mathrm{m}^{2}$. To study the optical properties of ZnSe nanowires, the cathodoluminescence (CL) spectrum was carried out using a temperature-controllable cathodoluminescence detector (Mono CL, Gatan) installed in the FE-SEM, which was performed at an accelerating voltage of $10 \mathrm{kV}$ and the probe current of $20 \mathrm{nA}$. The areas under CL measurement were about $40 \mu \mathrm{m} \times 40 \mu \mathrm{m}$. The CL detector enables measurements of the CL spectra between 200 and $850 \mathrm{~nm}$. 


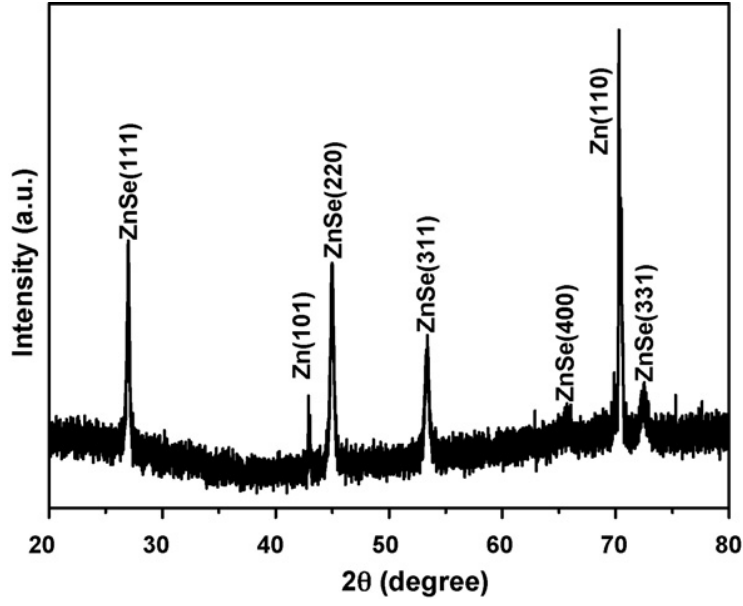

Fig. 1. X-ray diffraction pattern of as-grown ZnSe nanowires.

\section{Results and discussion}

The ZnSe nanowires were characterized by XRD to confirm the crystalline phase of the nanowires. Fig. 1 shows the representative XRD pattern of ZnSe nanowires synthesized at $400^{\circ} \mathrm{C}$ revealing cubic zinc blende phase of $\mathrm{ZnSe}$ with a lattice constant of $a=0.56 \mathrm{~nm}$, which matches well with those in JCPDS (Joint Committee on Powder Diffraction Standards, Card No. 37-1463). Zn signals in the XRD pattern were original from the uncovered portion of the $\mathrm{Zn}$ foil. The sharp XRD peaks imply good crystallinity. The chemical composition and stoichiometry of the ZnSe nanowires were investigated through EDX. The EDX spectrum recorded (not shown here) on a single $\mathrm{ZnSe}$ nanowire reveals the presence of only $\mathrm{Zn}$ and Se as the elementary components in the nanowire. Quantitative analysis shows that the atomic ratio of $\mathrm{Zn}$ to Se is very close to $1: 1$ stoichiometry.

Fig. 2a shows the general morphology, observed by scanning electron microscopy (SEM), and reveals that the deposited $\mathrm{ZnSe}$ nanowires were vertically aligned and grown in high density on the entire surface of the zinc foil. The average density of the nanowires was about $10^{9} \mathrm{~cm}^{-2}$. The typical diameters and lengths of the nanowires are in the range of $50-150 \mathrm{~nm}$ and $1-10 \mu \mathrm{m}$, respectively. Fig. 2b is a magnified SEM image of ZnSe nanowires, indicating that there are spherical particles terminating at the ends of the nanowires. The existence of spherical particles at the tip of nanowires provides a firm evidence for the self-catalytic liquid-solid mechanism [13].

On the basis of our experimental data presented here, the growth mechanism of the ZnSe nanowires were proposed as follows. Since there are no nanosized transition-metal or noble-metal catalyst particles introduced in our reactants, which are usually found to attach to or embed in the ends of the nanostructures fabricated by conventional VLS methods. Spherical particles can be clearly observed at the tip of the nanowires in our product. Therefore, the formation of $\mathrm{ZnSe}$ nanowires in our experiments suggests a self-catalytic liquid-solid mechanism proposed by Dang et al. [13], contrasting with the conventional VLS growth. The growth of ZnSe nanowires can be separated into four steps: (i) formation of nanoscale $\mathrm{Zn}$ droplets on the $\mathrm{Zn}$ surface at temperature close to its melting point, (ii) dissolution of further zinc and selenium vapour species into molten $\mathrm{Zn}$, (iii) phase segregation to create multiple nuclei on the substrate, and (iv) homoepitexial growth of these nuclei into one-dimensional structure [14].

To further explore the crystalline quality of the ZnSe nanowires, a microscopic confocal Raman spectrometer was used to obtain the Raman data of $\mathrm{ZnSe}$ nanostructures by focusing the laser fac-

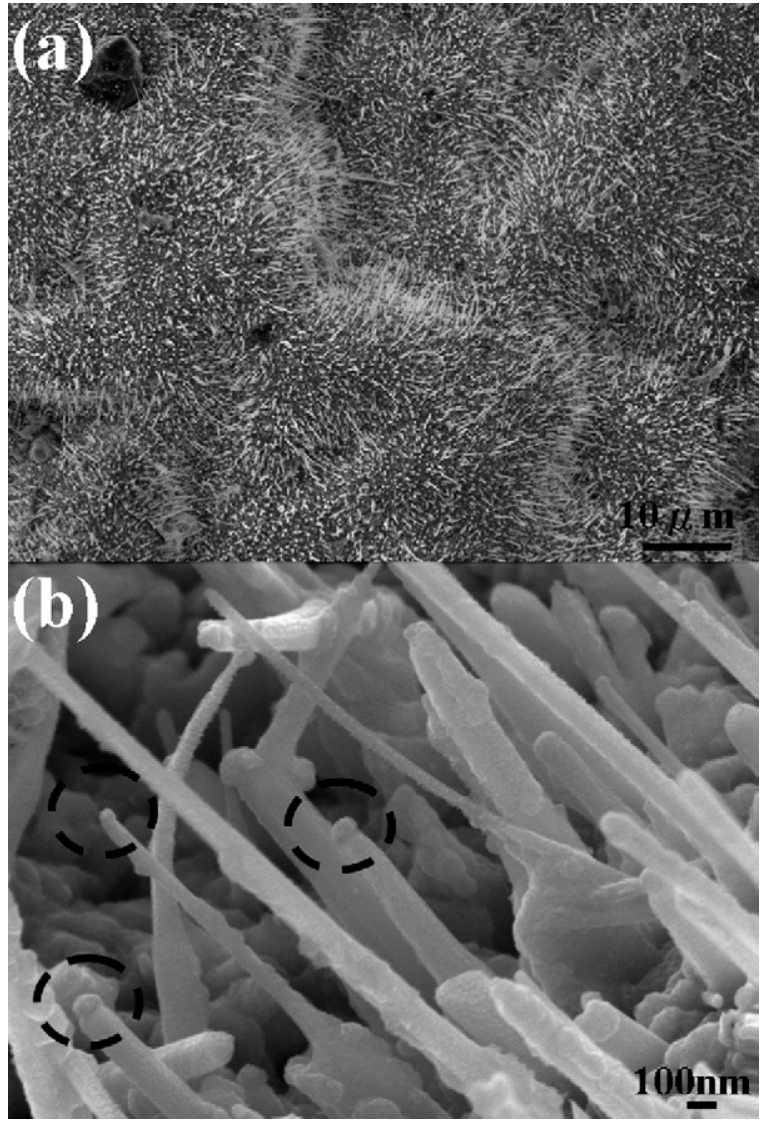

Fig. 2. (a) Low and (b) high magnification scanning electron microscope (SEM) images of vertically aligned $\mathrm{ZnSe}$ nanowires grown on zinc substrate.

ula on different locations. It was found that different areas show similar Raman profiles. Fig. 3 shows the typical Raman spectrum of a high purity sample. Two Raman peaks at 203 and $250 \mathrm{~cm}^{-1}$ can be attributed to the transverse optic (TO) and longitudinal optic (LO) phonon modes of ZnSe, respectively. No vibration modes due to impurities are observed. From previous reports [15-17], the LO phonon frequency of single-crystalline $\mathrm{ZnSe}$ is $255 \mathrm{~cm}^{-1}$ at room temperature. For ZnSe polycrystalline nanoparticles, the TO and LO phonon frequencies are 210 and $255 \mathrm{~cm}^{-1}$, respectively, and the broad Raman peaks may be attributed to the high surface-tovolume ratio of small particles. Compared with the previous results,

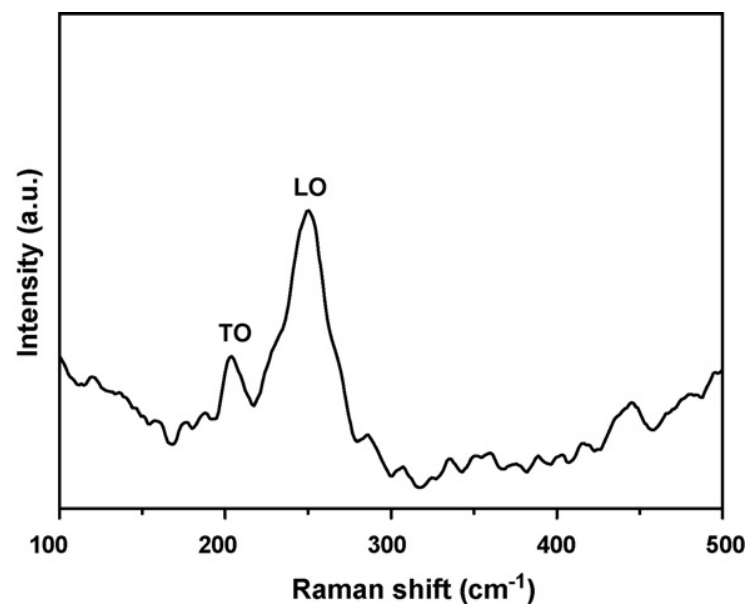

Fig. 3. Raman scattering spectrum acquired from $\mathrm{ZnSe}$ nanowires at room temperature. 


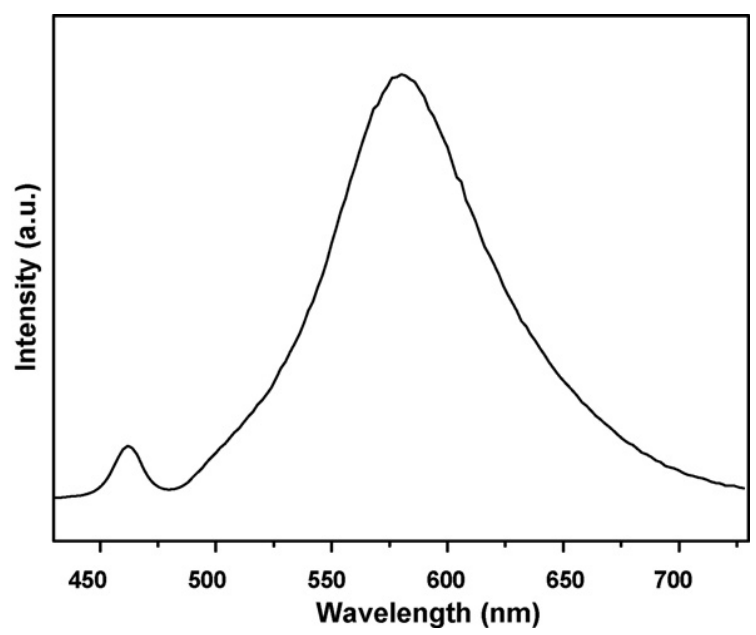

Fig. 4. Cathodoluminescence spectrum of ZnSe nanowires.

the LO and TO phonon peaks of the ZnSe nanowires are both shifted toward lower frequencies, which are probably due to the effects of small size and high surface area.

To investigate the optical properties of the ZnSe nanowires, the $\mathrm{CL}$ measurement was conducted at an acceleration voltage of $10 \mathrm{kV}$. Fig. 4 shows the typical room temperature CL spectrum of the as-grown nanowires. Generally, two bands appeared in the CL spectrum of the ZnSe nanowires, a weak narrow band at $462 \mathrm{~nm}$ and a strong broad band at $580 \mathrm{~nm}$. The weak narrow band is due to the recombination of free excitons through an exciton-exciton collision process also known as near-band-edge (NBE) emission and the visible deep-level-emission is because of the existence of nonstoichiometric defects, dislocations, and/or stacking faults $[2,18,19]$. Compared with the luminescence spectra of $\mathrm{ZnSe}$ nanostructures reported previously $[2,3]$, we found that most of the spectra only contain a broad defect emission and the band edge emission is hardly observed. This behavior reflects good crystalline quality of our grown $\mathrm{ZnSe}$ nanowires.

\section{Conclusions}

In summary, cubic zinc blende ZnSe nanowires have been synthesized on the zinc foil by thermal evaporation of selenium powder without the use of additional metal catalysts or additives at low temperature. General morphology of the synthesized $\mathrm{ZnSe}$ nanowires indicated that the grown products have diameter in the range of 50-150 $\mathrm{nm}$ with lengths of $1-10 \mu \mathrm{m}$. Raman scattering and room temperature CL spectra substantiated that the synthesized ZnSe nanowires are good in crystal quality with a zinc blende cubic phase containing few structural defects and exhibiting good optical properties. The obtained ZnSe nanowires therefore are highly favorable for the fabrication of optoelectronic devices due to their good crystallinity and visible luminescence properties and low production cost.

\section{Acknowledgements}

This work was supported by the Ministry of Education and National Science Council of the Republic of China.

\section{References}

[1] Z. Liu, S.K. Hark, Nanotechnology 17 (2006) 1355

[2] Y. Jiang, X.M. Meng, W.C. Yiu, J. Liu, J.X. Ding, C.S. Lee, S.T. Lee, J. Phys. Chem. B 108 (2004) 2784.

[3] L. Jin, W.C.H. Choy, Y.P. Leung, T.I. Yuk, H.C. Ong, J.B. Wang, J. Appl. Phys. 102 (2007) 044302.

[4] Y.P. Leung, W.C.H. Choy, I. Markov, G.K.H. Pang, H.C. Ong, T.I. Yuk, Appl. Phys. Lett. 88 (2006) 183110.

[5] S.V. Pol, V.G. Pol, J.M. Calderon-Moreno, S. Cheylan, A. Gedanken, Langmuir 24 (2008) 10462.

[6] X.T. Zhang, Z. Liu, Y.P. Leung, Q. Li, S.K. Hark, Appl. Phys. Lett. 83 (2003) 5533.

[7] C. Ye, X. Fang, Y. Wang, P. Yan, J. Zhao, L. Zhang, Appl. Phys. A 79 (2004) 113.

[8] D.Y. Xia, L. Dai, W.J. Xu, L.P. You, B.R. Zhang, G.Z. Ran, G.G. Qin, Chin. Phys. Lett 23 (2006) 1317.

[9] C. Jiang, W. Zhang, G. Zou, W. Yu, Y. Qian, Nanotechnology 16 (2005) 551.

[10] S. Ramanathan, S. Patibandla, S. Bandyopadhyay, J. Anderson, J.D. Edwards, Nanotechnology 19 (2008) 195601.

[11] U. Philipose, P. Sun, T. Xu, H.E. Ruda, L. Yang, K.L. Kavanagh, J. Appl. Phys. 101 (2007) 014326.

[12] Y.Q. Wang, U. Philipose, T. Xu, H.E. Ruda, K.L. Kavanagh, Semicond. Sci. Technol. 22 (2007) 175.

[13] H.Y. Dang, J. Wang, S.S. Fan, Nanotechnology 14 (2003) 738.

[14] S. Biswas, T. Ghoshal, S. Kar, S. Chakrabarti, S. Chaudhuri, Cryst. Growth Des. 8 (2008) 2171.

[15] T.J. Mountaiaris, J.D. Peck, S. Stoltz, W.Y. Yu, A. Petrou, P.G. Mattocks, Appl. Phys Lett. 68 (1996) 2270.

[16] B. Schreder, A. Materny, W. Kiefer, G. Bacher, A. Forchel, G.J. Landwehr, Raman Spectrosc. 31 (2000) 959.

[17] D. Sarigiannis, J.D. Peck, G. Kioseoglou, A. Petrou, T.J. Mountziaris, Appl. Phys. Lett. 80 (2002) 4024

[18] Y.C. Zhu, Y. Bando, Chem. Phys. Lett. 377 (2003) 367.

[19] X.T. Zhang, Z. Liu, K.M. Ip, Y.P. Leung, Q. Li, S.K. Hark, J. Appl. Phys. 95 (2004) 5752. 